\title{
A força da gravidade e a origem do movimento
}

\author{
Fernando Crêspo \\ Escola Superior de Dança, \\ Instituto Politécnico de Lisboa, Portugal
}

DOI: https://doi.org/10.31492/2184-2043.RILP2020.38/pp.81-99

\begin{abstract}
Resumo
No centro da ação performativa, vamos encontrar a relação que o corpo estabelece com a força da gravidade. A resistência ou entrega a esta força, a exploração do seu poder sobre o movimento e a presença da ilusão gravitacional noutras produções artísticas multidisciplinares, justificam este estudo. Aprofundam-se os momentos em que o corpo se ergue, movimenta ou é capaz de se orientar, precisamente, quando se opõe à força da gravidade, quando negoceia com esta ou quando se proprioceciona. Na variedade de superficies de suporte - às qual o corpo se apoia, agarra, pendura, salta e desliza - encontra-se um potencial criativo que resulta da relação de resistência à força da gravidade que o corpo estabelece, num determinado momento, com estas mesmas superfícies. Apresentam-se, também, meios circundantes que transformam, de forma radical, o movimento e sua relação com a gravidade. Por fim, retorna-se ao corpo, ao centro do corpo, para aí encontrar o impulso primordial do movimento.
\end{abstract}

Palavras-chave: alentejano; força da gravidade; resistência e entrega; superfície de suporte; meio circundante; centro do corpo.

\section{Abstract}

In the center of a performing action we find the relation established between body and gravity force. The resistance or surrender to this force, the investigation of its power over movement and the presence of gravitational illusion in other multidisciplinary artistic productions are the reasons for this study/reflection. We will explore the moments when the body raises and moves or find orientation, precisely when it resists gravity, when it negotiates with the force or when it becomes aware of its own proprioception. In the diversity of support surfaces - where the body rests, grabs, hangs, jumps or slides - we find a creative potential resulting from the relation of resistance towards the gravity force established by the body with these surfaces, in a precise moment. We will also present the surrounding means that have the potential to radically transform movement and its relation with gravity. Finally, we will return to the body and its core where we will find movement's primordial impulse.

Keywords: gravity force; resistance and yielding; supportative medium; surrounding medium; body center.

\section{Introdução}

A maneira como a arte tem lidado com a força da gravidade encontra-se refletida em diversos trabalhos, nos mais variados domínios artísticos. Enquanto que nas artes performativas a força da gravidade é um guião imposto ao movimento, em outras artes, pela natureza dos seus media, é possível fixar momentos em que a realidade, captada ou concebida pela relação que os corpos, objetos ou elementos

1. Este trabalho resulta da reorganização e desenvolvimento de parte da tese de doutoramento em Belas-Artes, especialidade Arte Pública (não publicada) “As ações do corpo no campo da coreologia e da produção artística multidisciplinar" - FBA-UL (2016). 
criam com a força da gravidade, transmite uma ambiguidade de situações em que a queda e levitação ${ }^{2}$ se tornam indistintas.

Como origem do movimento, procura-se encontrar a relação que o corpo mantém continuamente com a força de gravidade, numa expressão de resistência e entrega.

Aborda-se a forma como as técnicas de dança selecionadas fazem uso da força da gravidade, quer quando em desequilíbrio de um corpo, quer quando se encontra livremente em queda, quer ainda numa oposição clara e reforçadora da posição de equilíbrio.

As sensações musculares, provocadas pelos efeitos da força de gravidade sobre o corpo, vão-se encontrar como originadoras do sentido de orientação, sendo a vertical ao chão um referencial imutável, um eixo sobre o qual todas as outras direções são construídas.

Vai-se afirmar que, implícito na relação com a força da gravidade, se encontra o chão.

Dão-se exemplos de várias superfícies, variando em rigidez e aderência e em posicionamento espacial em relação ao corpo que suportam ou sustentam.

Avança-se com a identificação de um outro elemento, o do meio circundante (Crespo, 1988), que, em contextos teatrais, performativos ou naturais, transforma e influencia o corpo e o seu movimento.

Nos exemplos recolhidos de intervenção cénica sobre a superfície do palco, encontram-se soluções que revelam a interferência deste elemento no movimento dos corpos.

Considera-se um centro do corpo que - concentrado ou expandido, ponto de equilíbrio ou palco dinâmico de forças diversas, centro do corpo, centro de gravidade ou plexus solar - é um centro que, no corpo, cria e impele o movimento.

\section{Exploração Gravitacional}

A maneira como a dança tem lidado com a força da gravidade e igualmente com o chão, fronteira, limite desta relação, apresenta-se, por um lado, num campo de criação limitado por uma oposição total à força da gravidade, num distanciamento ao chão e, por outro, numa total entrega à força da gravidade, numa aproximação máxima ao chão.

No primeiro caso, em oposição à força da gravidade e num distanciamento ao chão, encontra-se um corpo expansivo de leveza, sem peso, "a sylphlike creature [sem qualquer evidência de uma] negotiation with gravity" (Albright, 1997, p. 56),

2. Levitação - entendida aqui como a ilusão criada pelas obras convocadas neste estudo, em que um corpo ou objeto se sustenta no espaço, sem ponto (aparente) de apoio, numa total oposição à lei da gravidade. 
uma excecionalidade às leis da gravidade que, de acordo com Jowitt (2010), se enquadrava perfeitamente na imagem de bailarina romântica e da "artist-as-rebel" (p. 215), numa atitude que demonstra não se encontrar enquadrada pelas leis que governavam a condição humana.

No segundo caso, na dança moderna, o corpo passa a interagir numa relação dinâmica com a força da gravidade. Designado muitas vezes por terrestre, isto é, "floor-bound and inward-looking" (Dempster, 2010, p. 230), o movimento apresenta uma ligação à terra, em oposição à verticalidade aérea; uma direção para dentro, em oposição à abertura e expansão espacial e cristalina das formas e posturas do ballet; um privilegiar do movimento sobre as formas/posturas, uma expressão provocada pela relação com a gravidade.

Numa clara oposição ao vocabulário do ballet, Isadora Duncan privilegia os movimentos de acordo com as disposições naturais do corpo, numa rejeição que considerou de artificial e de 'orthopedically unhealthy"' (Copeland, 2004, p. 64). Um dos elementos centrais que Isadora Duncan critica em relação ao ballet é, justamente, a relação com a força da gravidade ou com a lei da gravidade, como a própria refere: "All the movements of our modern ballet school $[\ldots]$ are unnatural: their purpose is to create the delusion that the law of gravitation does not exist for them.” (Duncan, 1977, p. 55). A sua posição teórica não possui, no entanto, um reflexo significativo nas práticas por si desenvolvidas, como refere Copeland (2004): "in practice, much of Duncan's dancing remained sprightly and vertical" (p. 64). A presença expressiva da relação com a força da gravidade - e de uma maior e mais evidente relação do corpo com a superfície de apoio, a terra, o chão - revela-se muito mais em Mary Wigman, Doris Humphrey e Martha Graham.

Segundo Copeland (2004), foi preciso esperar por Martha Graham para que a "modern dance truly came to terms with gravity and declared its determination to 'get down"'(p. 64).

No entanto, nos finais de 1940, quando estava mais que afirmada a presença elementar e natural da força da gravidade nas criações coreográficas da altura, Merce Cunnigham voltava a distanciar-se do solo, a elevar-se e a ocultar, expressivamente, a força da gravidade sobre os corpos em movimento, "he was busy cultivating his lightness, uprightness, and speed" (Copeland, 2004, p. 64).

Mas é com a técnica de contact improvisation que se torna visível, assumindo-se como central de todo o movimento, a presença de um "corpo responsivo" based in the physical exchange of weight" (Novack, 1990, p. 186). Um corpo que reage e enfatiza a entrega do seu peso quer ao chão, quer a uma outra superfície, a do corpo do outro, muito mais complexa e reativa. 
Há, assim, um abandono das formas, posturas e posições que são substituídas pela experiência e expressividade das sensações internas do fluxo do movimento entre dois corpos. É nesse ponto de contacto entre corpos que os impulsos, peso e momentum são comunicados, num contínuo envolvimento entre as forças que fluem nos corpos dos intérpretes. Um partilhar do peso e da superfície de suporte de cada um, como sublinha Albright (1997) "Contact Improvisation focuses on the physical relationship of one body to another, emphasizing the kinesthetic sensations and physics of weight and momentum rather than the visual picture of bodily shape within the stage space" (p.87).

Trisha Brown (1936-2017), recorrendo ao equipamento de montanhismo e de escalada (cordas, polias, faixas, cabos, roldanas, ganchos de pendurar), começa, em 1968, a explorar a suspensão dos corpos dos intérpretes e a sua deslocação sobre superfícies não horizontais. Em Man Walking Down the Side of a Building (1970), uma das coreografias do designado conjunto de 'Equipment Pieces' os performers suspensos, através da utilização do equipamento anteriormente referenciado, conseguem caminhar verticalmente às paredes do edifício, criando a ilusão de uma nova direcionalidade da força da gravidade. Parece existir, desta forma, uma força de gravidade que se exerce na direção vertical à parede, por onde os intérpretes se deslocam e, consequentemente, paralela à superfície, onde os espectadores se localizam. Esta facilidade dos corpos em deslocação horizontal e vertical cria uma renovada ilusão da ausência da força da gravidade, uma proeza 'sobrenatural', como refere Banes (1987). Embora não se veja a força da gravidade per se, vemos a sua atuação e é expectável que esta exerça uma força que os puxa para baixo, para o centro da terra sobre os objetos e corpos à superfície terrestre.

Algo semelhante acontece na cena do filme Royal Wedding de Stanley Donen (1951) em que Fred Astaire dança no chão, nas paredes e no tecto, o que vem a ser retomado nos anos 80 por Lionel Richie, em Dancing On The Ceiling (1986, Vídeo clip). Mas a verdade é que, nestes casos, não há qualquer desafio à gravidade por parte dos dançarinos. Em vez disso é, de facto, a sala que roda, mediante a criação de uma estrutura metálica que a transforma num engenho móvel, sugerindo, no olhar fixo da câmara, a alteração da posição dos corpos perante a gravidade.

Nos trabalhos de Mariko Mori ${ }^{3}$,Esoteric Cosmos (1996-98) e Burning Desire (1998), entre outros, podemos observar como a força da gravidade é desafiada sem, no entanto, criar algum estado de perturbação ou de tensão no observador. Antes pelo contrário, os ambientes criados por Mori, com figuras flutuando

3. Mariko Mori (Japão, 1967), fotografia, vídeo, escultura, instalação, arquitetura. 
em levitação, criam uma tranquilidade e transcendência, "an eclectic fantasy, a quirky dream-world" (Bailey, 2012, p. 340).

Os trabalhos de luz de James Turrel ${ }^{4}$ não só desafiam a gravidade como "they make physical earthen weight evaporate into the energy of light" (Kolodziej, 2012, p. 297). As suas esculturas de luz produzem uma ilusão ótica, criando a aparência de objetos físicos em levitação.

Em Leap into the Void (1960), Yves Klein ${ }^{5}$ coloca-nos perante uma imagem contraditória, a começar pelo enigmático título que pretende contextualizar este salto num vazio, um espaço sem referencial de uma base ou de um teto. No entanto, a rua e o céu que aparecem na fotografia, acabam por criar este referencial. Há um corpo que, simultaneamente, cai numa imposição inerente da força da gravidade e, por outro lado, a sua expressão de braços abertos, projetando-se para cima, para o céu, parecendo flutuar, "the moment where levity and gravity meet" (Kolodziej, 2012, p. 293).

Nos trabalhos de Kerry Skarbakka ${ }^{6}$, encontra-se, precisamente, a captação destes momentos, em que não se sabe se o corpo está num processo descendente ou ascendente. Embora numa situação de instabilidade pela perda de equilíbrio ou da sua base de sustentação, "the unknown outcome of his action" (Dzenko, 2012, p. 328), cria uma narrativa ambígua em que não se sabe se chegará ao chão em segurança ou terá um final trágico.

A gravidade torna-se assim um guião imposto ao movimento, uma força contrária ao espírito de todas as possibilidades da performance art, o expectável obrigatório.

\section{A Força da Gravidade}

Nas múltiplas relações que o corpo cria ao longo da sua vida, num esforço, curiosidade e reforço da sua presença e domínio, a força da gravidade surge como a relação primordial. Encontrando-se esta relação intermediada pelo meio envolvente - que circunda o corpo e a superfície de suporte - o chão ou outra superfície em que o corpo se apoia, agarra ou suspende. Nesta relação é possível observar, "two physical alignments" (Kaltenbrunner, 2004, p. 49), em resistência à força da gravidade. Uma resistência que iguala essa mesma força, impedindo qualquer movimentação ou recolhendo energia suficiente, como refere Zollig (2010), para superar a força que é exercida sobre si, possibilitando, assim, erguer-se e movimentar-se. Nesta movimentação, se a força da gravidade não for contrariada, cria

\footnotetext{
4. James Turrel (EUA, 1943), escultura, instalação, arquitetura, Land Art.

5. Yves Klein (França, 1928-1962), pintura, performance art.

6. Kerry Skarbakka (EUA, 1970), fotografia, performance art.
} 
um movimento de desequilíbrio ou de queda com a duração precisa do período em que o corpo se entrega até voltar a resistir, adiando a queda ou amortecendo-a no chão.

\subsection{Erguer}

A postura erguida que caracteriza a espécie humana é a evidência viva de uma controlada oposição à força da gravidade, uma ação que permanece sensivelmente constante ao longo da vida.

Feldenkrais (1987) chega mesmo a atribuir à força da gravidade - enquanto manifestação primeira de uma força exterior ao corpo, com que este se depara e à qual resiste - a responsabilidade no despertar para a vida sensível e pensante. Uma ativação que se inicia, precisamente, no momento em que uma miríade de conexões nervosas se alinham num movimento de resistência a esta força, "to maintain the body against the pull of gravity" (p. 33).

Numa clara oposição à força da gravidade encontra-se o momento em que o corpo se levanta, assumindo uma postura erguida, organizando-se e desenhando-se singularmente. Para Feldenkrais, esta postura torna-se aceitável quando não entra em conflito com a lei da natureza, em que o sistema nervoso realiza, sob a influência da gravidade, os ajustes necessários no esqueleto para que este sustente o corpo, usando uma energia mínima, isto é, uma postura em que a estrutura óssea, o esqueleto, consiga neutralizar o "pull of gravity, leaving the muscles free for movement" (Feldenkrais, 1987, p. 67).

Numa outra perspectiva, Straus (1966) afirma que a permanência de uma força em resistência à força da gravidade revela que é próprio da natureza humana opor-se, com os seus meios naturais, à natureza nos seus aspetos fundamentais. Isto é, uma oposição à força da gravidade, uma ação contrária a esta, mas que simultaneamente estabelece uma integração com o mundo no momento em que procura separar-se deste, "Much as we are part of nature with every breath, with every bite, with every step, we first become our true selves in waking opposition to nature." (p.168).

\subsection{Movimentar}

A atração gravitacional que a massa terrestre exerce sobre a massa do corpo, uma força que atua verticalmente para baixo, confere ao corpo o seu peso. $\mathrm{O}$ peso de um corpo é, pois, a força com que a Terra o atrai.

$\mathrm{Na}$ Countertechnique - uma técnica que faz uso das "directions and counter directions in both the body and space" (Siegmund \& van Dijk, 2014, p. 67) - encontra-se, precisamente, a utilização sistemática do peso do corpo ou do peso de 
uma parte do corpo, para a criação do movimento. É precisamente quando o peso do corpo cai fora da sua base de sustentação que "becomes available for movement" (p. 68), exercendo-se sobre este potencial movimento, ativado pela força da gravidade, todo um conjunto de forças contrárias de modo a permitir uma liberdade e controlo do movimento assim criado. Um momento de estabilidade resultante da composição das forças criadas em oposição ao movimento de queda ou de desequilíbrio, um controlo sobre o peso, com o seu aproveitamento para a criação de movimento.

De modo semelhante, encontra-se, na técnica de Humphrey/Limón, no swing 'fall and recovery', a criação deste momento: um movimento que integra a velocidade criada pelo movimento pendular descendente para, em continuidade, criar o balanço no movimento ascendente.

Mas é na técnica de Contact Improvisation que a força da gravidade, exercida sobre a massa do corpo e o peso deste sobre a superfície que o suporta, se torna expressivamente visível. Como refere Kaltenbrunner (2004) "Gravity gives impetus for movement" (p. 52), logo que a superfície de contacto desaparece, tal como na ação de cair, em movimento aéreo ou numa relação sem resistência ao chão, como no caso de desfalecer, ou tende a desaparecer, como no caso de desequilibrar, "we become a precarious victim of gravity" (p. 52).

A partir deste instante, com maior ou menor grau de entrega à força da gravidade, o corpo tem a possibilidade de utilizar este impulso gerado, esta dinâmica assim criada, para encontrar novas direções para o movimento. A utilização deste momentum, central nesta técnica, possibilita ainda reduzir a energia muscular ao mínimo, uma vez que o movimento é criado e mantido, embora mais ou menos controlado, como referido anteriormente, pela força gravitacional exercida sobre a massa do corpo.

Numa utilização diferenciada, encontram-se as abordagens técnicas da dança clássica que recorrem, precisamente, à força que o corpo exerce sobre a superfície que o suporta, a força que atravessa toda a massa do corpo ao longo do eixo central, para reforçar o equilíbrio das posturas recorrentemente utilizadas.

Para Tözeren (2000), a força da gravidade atua positivamente se a projeção da força no corpo for coincidente com a direção da deslocação do mesmo, como nas técnicas de Countertechnique e Contact Improvisation, "When a particle falls toward earth, gravity does positive work on the particle" (p. 65). Por outro lado, quando um corpo se desloca na vertical (erguer ou saltar, por exemplo), o trabalho realizado pela gravidade é negativo, opondo-se desta maneira à direção da força e da trajetória do corpo. 
Analisando a ação de pausar, observa-se aí uma resistência à força da gravidade sem, no entanto, se verificar qualquer deslocação, total ou parcial, do corpo no espaço. Este momento estático do corpo requere, porém, um gasto calórico necessário à sustentação do seu peso. Podemos, então, considerar, em complemento ao raciocínio de Tözeren, que a força da gravidade não atua positivamente, impulsionando o movimento, nem negativamente, atuando no sentido contrário ao movimento. A força da gravidade é anulada pela força que impede o corpo de se mover, sendo considerada aqui de valor nulo.

\subsection{Orientar}

A força da gravidade, ao obrigar a uma constante equipendência entre resistência e cedência, acaba por criar, de acordo com Juhan (1998), todo um conjunto de sensações musculares que geram os mecanismos usados para sentir e controlar este equilíbrio, o que leva este autor a concluir que "our very concept of space itself is primarily muscular” (p. 249). É, justamente, por isso que a percepção desta força cria, na sentida verticalidade ao chão, a estrutura das direções espaciais. Imutável na sua direcionalidade gravitacional, o eixo vertical é a referência primeira sobre a qual os outros eixos espaciais se dimensionam: um eixo vertical, onde as direções cima e baixo existem numa oposição total ou cedência completa à força da gravidade; e outro, numa manutenção constante de resistência equilibrada a essa mesma força, cria uma horizontalidade, uma perpendicularidade à força da gravidade, numa explosão de direções possíveis num plano horizontal, tornando-se, nestas circunstâncias, as direções frente/trás e direita/esquerda, estruturantes da orientação.

Nas direções criadas, dentro do sistema de Notação Laban (Labanotation ou Kinetography Laban), faz-se uso deste mesmo sistema referencial de orientação, onde os eixos da "standart cross of axes" (Hutchison, 1977, p. 415), por exemplo, se encontram alinhados com a direção da gravidade na definição da direção cima/ baixo, recorrendo-se ao corpo para definir as restantes direções: a frente é a sua frente e assim sucessivamente.

A perceção que o corpo possui da direção da força da gravidade só é possível porque este se encontra dotado de um sistema proprioceptivo "that synthesized information about joint positioning, muscular exertion, and orientation within space and with respect to gravity" (Foster, 2011, p. 44). A proprioceção permite pois detetar a força da gravidade e, desta forma, de pé, sentados ou deitados, sabe-se qual é a orientação relativamente à gravidade. Todas as outras direções são, pois, construídas tendo como matriz esta força sentida verticalmente ao chão, eixo perpendicular ao plano da terra, um imutável quadro de referência "the earth's gravitational field" (Sparshott, 1995, p. 118). 


\section{Limitações e Possibilidades}

\subsection{A superfície de suporte}

Sem o chão, sem uma superfície onde o corpo se pudesse apoiar, agarrar ou suspender, o corpo afundar-se-ia nas profundezas da massa terrestre. Sem a aderência proporcionada por uma superfície, o corpo não teria onde encontrar resistência para criar a fricção indispensável à deslocação. Sem a estabilidade de uma superfície, o corpo não tinha como se manter equilibrado. Sem uma superfície suficientemente rígida, o corpo não teria como se empurrar numa projeção aérea de um salto.

As superfícies que dão suporte ao corpo são, pois, um elemento determinante nas características do movimento criado em contacto com elas. Variam em rigidez, influenciando os movimentos na vertical. E em aderência, afetando os movimentos na horizontal. Apresentam-se em diversas relações espaciais com o corpo: por baixo, como o chão que o suporta; por cima, como a trave que permite a suspensão; pelos lados, como a barra ou como a corda que possibilita deslocações na vertical.

Habitualmente o corpo utiliza elementos sólidos para se apoiar em resistência à força da gravidade. O chão, extensa superfície percorrida pelo corpo, suporte da deslocação espacial, é um elemento constante. Cadeiras, cordas, barras, varões que possam suportar, suspender ou auxiliar numa deslocação vertical, são alguns outros materiais, dispostos espacialmente, com os quais o corpo, potencialmente, se relaciona. Esta relação que envolve uma clara transferência do peso do corpo para essa superfície, palco da relação de forças em ação, é realizada sempre por uma parte ou um conjunto de partes do corpo: as nádegas e costas sobre a cadeira; os tornozelos presos numa corda; um mastro vertical circense que as mãos agarram e as pernas envolvem. Seja no equilíbrio de um pé em pontas, de duas mãos num pino, da cabeça na pirueta do breakdance ou de várias superfícies dos braços, pernas e tronco, quando deitado ou a rebolar, importa aqui considerar as variações de natureza das superfícies que dão apoio ao corpo, uma vez que acabam por interferir nas ações realizadas.

É na dança e nas artes circenses que as artes performativas evidenciam, com mais clareza, a importância que possuem as diversas superfícies ou elementos que, na performance pretendida, suportam o corpo: cordas onde o corpo se agarra e pendura, cordas que prendem ou possibilitam o deslizar do artista, cordas que, esticadas na horizontal ou em inclinação acentuada, desafiam a uma travessia em equilíbrio precário; mastro circense que enfrenta e provoca uma escalada, uma suspensão, um desenho do corpo paralelo ao chão e vertical a uma barra, também ela vertical; trapézios voadores, o suporte que se desloca e balança pelo ar e 
camas elásticas que ampliam a projeção aérea, são alguns exemplos da panóplia de recursos técnicos empregues no circo que transformam radicalmente o movimento dos artistas.

Na dança, o chão do palco tem sido objeto das mais diversas transformações. O som do movimento sobre a superfície de contacto tem sido manipulado, por exemplo, pela adição de materiais, como folhas em Bluebeard (1977) ou uma ligeira camada de água como em Arias (1979) - ambas obras de Pina Bauch que amplificam a relação do movimento sobre essa superfície quer através do farfalhar da folhagem, quer através do som dos salpicos da água, respetivamente. Em sentido contrário, qualquer som produzido pelo andar ou pelas corridas observáveis em 1980 - A piece by Pina Bausch (1980) é absorvido pela turfa que cobre inteiramente o chão do palco.

Os sistemas de notação do movimento, com o objetivo de registarem as deslocações dos bailarinos nas suas trajetórias pelo espaço, têm recorrido, desde o início do séc. XVII, a desenhos que revelam o percurso realizado pelos bailarinos dentro do tempo confinado a cada dança. Intricadas deslocações podem ser encontradas em Nobiltà di Dame de Fabritio Caroso, publicado em Veneza em 1600, ou em Chorégraphie, ou l'art de décrire la danse, Paris, 1700 - um percurso que, durante essa dança, regista num único desenho, toda a deslocação pela sala. Em Rite of Spring (1975) de Pina Bauch, o chão, ao ser coberto de terra, possibilita que a movimentação dos bailarinos fique aí inscrita, como pegada deixada para trás na terra. Desta maneira, zonas de forte atividade são reveladas nos sulcos profundos e torcidos, enquanto outras zonas mais calmas revelam unicamente simples traços.

Uma outra alteração da superfície de suporte é o exemplo apresentado em Raft Piece (1973), de Trisha Brown. Aqui os bailarinos apoiam-se em jangadas que flutuam sobre um lago, uma horizontalidade em permanente oscilação, um lugar para o desequilíbrio.

Mas a alteração mais radical da superfície de contacto é apresentada por Simon Forti em Fallers (1968), com a completa e total inexistência da superfície de suporte. Os espectadores, situados num andar, vêem, através da janela, os corpos que caem livremente.

\subsection{Meio circundante}

À superfície terrestre, o ar enquanto meio de natureza gasosa e que se encontra permanentemente a envolver e a tocar o corpo, não apresenta uma resistência ou fricção significativa. Ela até é, para o movimento realizado "indoors", de valor desprezível. No entanto, é precisamente este elemento natural que, quando 
agitado e colocado em movimento, exerce, consequentemente, uma influência sobre os corpos e objetos, podendo fazê-los deslocar-se ou, adicionando uma força a favor ou contra o movimento, dificultar ou auxiliar a deslocação do corpo ou partes deste.

No desporto, os valores de tempo e distância e ainda a escolha da direção do jogo, em função da direção do vento, representam a evidência desta força - uma força que, à superfície terrestre, ao nível do homem, se exerce na horizontal ou por reflexão na superfície terrestre, de baixo para cima, fazendo os corpos elevarem-se ou ainda, pelo encontro com outros obstáculos, mudar de direção.

Este mesmo meio erode e desenha sobre a terra o rasto da sua força à passagem. Obriga o corpo a inclinar-se na sua direção para não cair, para não ser derrubado, como um vento que anima os cabelos dos corpos e simultaneamente cola e desprende a roupa que o corpo veste. Este efeito que o ar em movimento potencialmente cria sobre os corpos, realiza-se a nível cinético, recompondo as posturas e a relação com a força da gravidade. Tornado (2007), de João Tabarra, é o exemplo, embora criado artificialmente, da força do ar quando em movimento. O artista é impossibilitado de entrar no seu estúdio (?) pela agitação criativa (?) que este contém: um tornado criado no interior empurra o performer contra as paredes e pela porta fora.

O fumo, o nevoeiro ou a poeira, adicionados ao ar, são, também eles, elementos que alteram o meio circundante e, consequentemente, a visibilidade do observador, sendo considerados 'special effects'. É curioso lembrar que no séc. XVII, o fumo que hoje se considera como um efeito especial deliberadamente usado no palco, estava sempre presente, sendo naturalmente produzido pelas fontes de luz da altura, velas, candeias a óleo que produziam um "mystical effect" (Streader \& Williams, 1985, p. 13).

Quando a composição do ar, nas suas proporções de nitrogénio, oxigénio, dióxido de carbono e vapor de água, é alterada pela introdução de outros elementos, como por exemplo, os referidos anteriormente - e embora as condições acústicas não se alterem significativamente - o campo visual e a sua composição sofrem importantes transformações. Podem tapar ou ocultar parcial ou completamente o cenário ou os corpos dos performers e os seus movimentos, "working as a kind of plastic ephemeral set or prop." (Crespo, 1988, p. 26). A sua plasticidade pode ser usada para modificar a forma do corpo, como em De noite, de Corsetti (1988) em que os atores/bailarinos se encontravam cobertos de poeira, o que alterava significativamente a sua forma, impossibilitando a distinção entre frente e trás do corpo, movimentando-se para um centro de forças magnéticas e metamorfoseando-se num corpo indistinto, expelindo poeira por todo o espaço numa indeterminável 
nuvem de movimento. A alteração deste medium resulta ainda de uma forma mais radical quando associado à iluminação de palco, revelando a fonte e enfatizando a direcionalidade da luz.

Com uma densidade diferente do ar, a água é um meio que, com as suas características de maleabilidade e transparência, se torna possível como um lugar de performance. Waterproff (1986), de Daniel Larrieu, é um exemplo paradigmático da exploração deste meio. Waterproff apresenta uma imaginativa variedade de situações em que a água, contida numa piscina, é o local onde as ações dos bailarinos se localizam.

Enquanto vídeo-dança, as câmaras encontram-se posicionadas de três formas: a primeira localizada dentro da água, a segunda fora da água mas permitindo uma visão dos bailarinos dentro, e a terceira fora da água mas, devido ao ângulo e à iluminação, os bailarinos que se encontram dentro de água ficam ocultos.

No primeiro caso podemos observar que estes corpos não se debatem com a força da gravidade, da mesma forma que o fazem fora deste meio. As descidas ao fundo da piscina realizam-se recorrendo ao esvaziamento dos pulmões ou a manutenção à superfície pelo enchimento pleno dos mesmos. Por outro lado, os movimentos, devido à densidade da água, tornam-se mais difíceis, parecendo estar a ser executados em permanente 'slow motion'.

No segundo caso e pela refração da luz, observamos uma alteração da forma do corpo e das suas partes, uma deformação que, umas vezes encolhe, outras vezes alonga os corpos. Este fenómeno ótico, associado à movimentação da água à superfície, cria nos corpos uma articulação múltipla de ações de torcer e contrair, localizadas por todo o corpo.

Por último, as entradas e saídas de cena, normalmente efetuadas pelos lados do palco, são possíveis de ser realizadas aqui, por debaixo do chão, ou seja, emergindo ou submergindo da linha de água à superfície.

\section{No centro do corpo}

\footnotetext{
"The center is not only the initiation point for all movement, it is also the driving force for all movements through the space." (Hardt and Sander, 2014, p. 252).
}

Embora sendo um conceito subjetivo, a definição e localização do centro do corpo, centro da força ou centro da gravidade, é abordada em diversos campos do conhecimento.

Num contexto do desenho arquitetónico e da sua relação com o corpo humano, Marcus Vitruvius (séc. I) situa o centro precisamente no umbigo do corpo, 
um ponto equidistante das extremidades dos seus membros, "naturally placed in the centre of the human body, and, if a man lying with the face upward, and his hands and feet extended, from his navel as the centre, a circle be described, it will touch his fingers and toes." (Vitruvius, 1826, p. 79). Este círculo assim desenhado, encontra-se exemplificado por Leonardo da Vinci naquilo que se tornou um dos desenhos mais célebres da representação humana, O Homem de Vitruvius. Um círculo e um quadrado, contendo no seu interior uma dupla representação humana que toca com as extremidades dos seus membros esticados, quer a circunferência quer os lados do quadrado; o centro é localizado na representação bidimensional de um corpo estático.

Encontra-se em Weaver (1721), talvez numa interpretação deste mesmo desenho, uma definição que se apoia na magnitude possível de um corpo - a extensão dos membros e as suas direções convergentes, representadas no referido desenho, ou seja, as linhas que irradiam de um ponto ou que para ele convergem - o "center of heavy bodies, or center of magnitude, is a certain point in the middle of that body, equally distant from its extremities as much as possible, and to which all its parts tend" (p. 97). Um centro em que todos os planos que pretendam dividir equilibradamente o corpo, terão obrigatoriamente de o atravessar.

O centro do corpo que, na sua relação com a força da gravidade, se designa como centro de gravidade, é um ponto a que os bailarinos se referem como o 'seu centro' e que se relaciona com o seu próprio equilíbrio. Como refere Hutchison (1977) é "that point in the body from which or on which the body can be suspended or poised in equilibrium" (p. 389). Trata-se de um ponto subjetivo para onde converge a força de gravidade que atua em todo o corpo. "We will define 'center of gravity' as that point where the downward force of gravity appears to act on the body as a whole" (Laws, 2002, p. 20) e que, de acordo com Kaltenbrunner (2004), reúne todas as forças que afetam o corpo. Uma linha imaginária que daí parta para o centro da terra, define o "gravitational axis" (p. 47). É um ponto que, de acordo com o mesmo autor, se situa perto do centro anatómico do corpo.

Embora sem localização precisa, como refere Kaltenbrunner (2004), e que numa "normal extended position" (p. 47) apresenta uma proximidade do centro de gravidade com o centro anatómico, a sua localização varia de acordo com a constituição física e posicionamento do corpo. Como refere Hutchison (1977) "its exact position depending on the build of the individual and on the position taken" (p. 389), assumindo-se aqui a variedade de posições como uma sequência contínua de movimentos, alterando, consequentemente, a localização do centro de gravidade que, não sendo estático, se reposiciona no corpo em movimento, "the position of the center of mass of a human body is not stationary but varies with body movement." (Tözeren, 2000, p. 56). 
Se, até ao momento, se tem encontrado neste 'centro' - localizado próximo do centro anatómico, do centro inicialmente idealizado por Vitruvius e, posteriormente, desenhado por Da Vinci - um ponto de concentração e de convergência da força da gravidade que atua em todo o corpo, ou seja, um ponto de equilíbrio, encontra-se, também aí, um palco em que diversas forças atuam - "point where a distributed force or a collection of several forces may be considered to act" (Laws, 2002, p. 25).

Mesmo na situação aparentemente estável de um equilíbrio, ao centro do corpo converge todo um conjunto de forças exteriores provenientes da força da gravidade, da relação com o chão e de forças internas que criam um "energized and stable center [...] required for playing with balance, for turns and tilt" (Hardt and Sander, 2014, p. 252).

A existência de um centro de gravidade que domina o movimento por si gerado é observada por Kleist em 1810, aludindo ao teatro das marionetas e ao seu funcionamento: "Cada movimento, [...] tinha um centro de gravidade; bastava portanto comandar esse centro" (Kleist, 2009, p. 134).

Wigman deslocou o 'center of kinetic energy' das pernas, coxas e quadris para o tronco, o que, segundo Toepfer, (1997), veio dramatizar "a struggle with gravity rather than an ethereal escape from it" (Toepfer, 1997, p. 111).

O centro do movimento, o 'solar plexus' como é designado por Duncan (1928), é a fonte impulsionadora do movimento e da dança, "le ressort central de tout mouvement, le miroir de vision d'où jaillit la danse, toute créée" (Duncan, 1928, p. 80). Daly (1995) reporta esta descoberta a Delsarte: "From Delsartism, she [Duncan] learned about the importance of the body's center (what she later identified as the solar plexus) as the source of physical expression" (p. 135). Para Delsarte, segundo Delaumosne (1893), o tronco é visto como um centro dinâmico, dividido "into the thoracic centre for the mind, into the epigastric for the soul, and into the abdominal for the life" (p. 109). Um 'vital centre' que Delsarte associa ao "centre of gravity" (Stebbins, 1887, p. 170).

Para Duncan, o 'solar plexus' era igualmente uma fonte de expressão e de revelação do espírito, existindo para além das suas manifestações materiais, irradiando pelos braços e pernas, projetando-se pelo espaço. "Her 'soul' awakened" (LaMothe, 2006, p. 121) era a fonte da expressão espiritual, da qual irradiavam, por todos os canais do corpo, "la force centrifuge qui reflète la vision de l'esprit" (Duncan, 1928, p. 80).

Em vez de isolado numa localização anatómica, Martha Graham expande este centro para toda a zona onde a respiração, o movimento de inspiração e expiração, acontece. Estiliza estes movimentos "into kinetic images [que a mesma designou 
de] 'contraction' and 'release"' (LaMothe, 2006, p. 172). A sua técnica, baseada na respiração e que Graham (1991) considera ser a pulsação da vida, é composta por estes movimentos básicos do corpo, dois movimentos que acompanham o homem e que serão mantidos desde o nascimento "until you die" (p. 46). Há uma inalação de vida em release', e uma 'contraction' que devolve esse mesmo pulsar de vida ao mundo, gerando uma energia que "animates the world and everything in it [...] It can be Buddha, it can be anything, it can be everything. It begins with breath" (Graham, 1991, p. 46).

Com esta visão, Graham não só expande a localização do centro criador do movimento para toda a área onde a respiração ocorre, como torna este mesmo centro dotado de uma dinâmica inerente, suportada pelo movimento da própria respiração. O melhor exemplo é-nos dado pela própria coreógrafa, referindo-se ao movimento de contração da seguinte forma: "contraction is not a position. It is a movement into something. It is like a pebble thrown into the water, which makes rippling circles when it hits the water" (Graham, 1991, pp. 250-251).

No corpo espacial e cristalográfico de Rudolf Laban, o centro é um ponto a partir do qual irradiam as direções, um centro coincidente com o centro do octaedro, em que os vértices indicam as direções, alinhando-se com os eixos do corpo. "The center of the octahedron coincides with the center of the moving body" (Maletic, 1987, p. 68).

Podemos encontrar em Laban uma ampliação do movimento de 'contration' e 'realese' de Graham, dois movimentos que têm por base uma mesma relação pulsante com o espaço e uma mobilização total do corpo. As ações primitivas de semear (scattering) e recolher (gathering) apresentam-se alternadamente numa sequência de movimentos "of scattering from the centre of the body outwards into the surrounding space [...] with the action of gathering from the periphery of the kinesphere inward towards the centre" (Maletic, 1987, p. 66).

Concentrado ou expandido, ponto de equilíbrio ou palco dinâmico de forças diversas, centro do corpo, centro de gravidade, plexus solar, o centro cria e impele o movimento.

\section{Conclusão}

Embora permanentemente impositiva, a força da gravidade tem sido constantemente desafiada em diversos domínios artísticos. Nas artes performativas e, particularmente, na dança, as variações da relação de resistência e entrega de um corpo à força da gravidade tem caracterizado épocas, géneros e estilos. O balletnuma oposição e distanciamento ao palco -, a dança moderna - em ligação à terra, determinada em descer numa verticalidade ao chão - ou, ainda, as abordagens 
contemporâneas como a técnica de contact improvisation countertechnique - em que se cria um corpo responsivo, capaz de capitalizar o momentum de entrega do seu peso ao chão e ao outro - são exemplos demonstrativos da relevante participação da força da gravidade na construção de técnicas e explorações artísticas variadas.

Mas, esta relação primordial proporciona ainda outras oportunidades de desenvolvimento e afirmação corporal:

- No momento em que se levanta e assume uma postura erguida, organizando-se e desenhando-se corporal e individualmente, numa ação clara de oposição à força da natureza. Nesse instante, segundo Feldenkrais (1987), cria-se uma miríade de conexões nervosas que faz despertar para a vida sensível e pensante. Neste encontro da força da gravidade com o corpo, há todo um mundo que o percorre e atravessa, que o mantém conectado à terra, como um elo dinâmico que mantém o corpo à pele da Terra e que a ela o faz pertencer;

- $\quad$ Nas técnicas de contact improvisation e countertechnique - que procuram e tornam visível a relação da gravidade sobre a massa do corpo e o peso deste sobre a superfície que o suporta - descobre-se a força da gravidade como impulsionadora do movimento;

- É possível encontrar a origem do sentido de orientação nas sensações musculares provocadas pelos efeitos da força de gravidade sobre o corpo, sendo a vertical ao chão um referencial imutável, um eixo sobre o qual todas as outras direções são construídas.

Como mediadores da relação com a gravidade identificou-se:

- $\quad$ O chão e outras superfícies de suporte que variam em rigidez, aderência e relação espacial com o corpo que nelas se apoia, agarra ou suspende. Estas variações têm sido amplamente exploradas na dança e nas artes circenses;

- O meio circundante, embora habitualmente não ofereça resistência, pode tornar-se uma força capaz de erodir a superfície terrestre e alterar a postura, a forma e o movimento do corpo - como o ar, quando colocado em movimento. Este e outros media, com as suas alterações estáticas e composicionais, interagem significativamente no movimento, na configuração dos corpos e na visibilidade dos mesmos.

Seja num desenho, como o Homem de Vitruvius de Da Vinci - em que o centro do corpo surge como um ponto equidistante dos seus membros estendidos -, seja um ponto, centro de magnitude, coincidente com todos os planos que pretendam 
dividir equilibradamente, em peso e força, o corpo humano - como em Weaver (1721) - ou no conceito de centro de gravidade, (Hutchison, 1977), (Laws, 2002), (Kaltenbrunner, 2004), (Hart, 2014) - como ponto de equilíbrio, em que a força da gravidade parece agir sobre o corpo como um todo, um centro estável, mas simultaneamente energético - é no centro do corpo que vamos encontrar a origem do movimento.

\section{Referências}

Albright, A. (1997). Choreographing difference: The body and identity in contemporary dance. Middletow: Wesleyan University Press.

Bailey, E. (2012). Mari Mori and Pipilotti Rist: Reflections on the "new levity" in arts. In M. Edwards \& E. Bailey (Eds.), Gravity in arts: Essays on weight and weightlessness in painting, sculpture and photography (pp. 338-341). Jefferson: MacFarland \& Company, Inc., Publishers.

Banes, S. (1987). Terpsichore in sneakers: Post-modern dance. Wesleyan: Wesleyan University Press.

Copeland, R. (2004). Merce Cunningham: The modernizing of modern dance. New York: Routledge.

Crespo, F. (1988). A justification for the inclusion of the strands of the dance medium in dance analysis. Master of Arts in Dance Studies - thesis. Laban Centre for Movement and Dance, London.

Daly, A. (1995). Done into dance: Isadora Duncan in America. Bloomington: Indiana University Press.

Delaumosne, L'Abbé (1893). Delaumosne on Delsatre. In Delsarte system of oratory (pp. xiii170). New York: Edgar S. Werner.

Dempster, E. (2010). Women writing the body: let's watch a little how she dances. In A. Carter and J. O'Shea (Eds.), The Routledge dance studies reader (pp. 229-235). Oxon: Routledge.

Duncan, I. (1928). Ma vie. Paris: Librairie Gallimard.

Dzenko, C. (2012). Photographic moments inside of gravity: Kerry Skarbkka's struggle to right himself. In M. Edwards \& E. Bailey (Eds.), Gravity in arts: Essays on weight and weightlessness in painting, sculpture and photography (pp. 326-337). Jefferson: MacFarland \& Company, Inc., Publishers.

Feldenkrais, M. (1987). Awareness through movement: Health exercises for personal growth. Harmondsworth: Penguin Books Ltd.

Foster, S. (2011). Choreographing Empathy. London: Routledge. iBooks.

Graham, M. (1991). Blood Memory. New York: Doubleday.

Hardt, Y. and Sander, V. (2014). Understanding the body/movement. In I. Diehl, F. Lampert (Eds.), Dance techniques 2012: Tanzplan Germany (pp. 250-255). Leipzig: Henschel Verlag in der Seemann Henschel GmbH \& Co. KG. 
Hutchison, A. (1977). Labanotation or Kinetography Laban: The system of analyzing and recording movement. New York: Theatre Arts Books.

Jowitt, D. (2010). In pursuit of the sylph: ballet in the Romantic period. In A. Carter and J. O'Shea (Eds.), The Routledge dance studies reader (pp.209-219). Oxon: Routledge.

Juhan, D. (1998). Job's Body. New York: Station Hill.

Kaltenbrunner, T. (2004). Contact improvisation: Moving - dancing - interaction. Oxford: Meyer und Meyer

Kleist, H. (2009). Sobre o teatro das marionetas e outros escritos. Lisboa: Antígona.

Kolodziej, M. (2012). From vertigo to ethereality in environmental art. In M. Edwards

\&. Bailey(Eds.), Gravity in arts: Essays on weight and weightlessness in painting, sculpture and photography (pp. 293-303). Jefferson: MacFarland \& Company, Inc., Publishers.

LaMothe, K. (2006). Nietzsche's Dancers: Isadora Duncan, Martha Graham, and the revaluation of Christian values. New York: Palgrave MacMilan.

Laws, K. (2002). Physics and the art of dance: Understanding movement. Oxford: Oxford University Press.

Maletic, V. (1987). Body-space-expression: The development of Rudolf Laban's movement and dance concepts. Berlin: Mouton de Gruyter.

Novack, C. (1990). Sharing the Dance: Contact Improvisation and American Culture. Madison: University of Wisconsin Press.

Siegmund, G. \& van Dijk, A. (2014). Introduction: The difficulty of running. In I. Diehl \& F. Lampert (Eds.), Dance techniques 2010: Tanzplan Germany (pp. 66-69). Leipzig: Henschel Verlag in der Seemann Henschel GmbH \& Co. KG.

Sparshott, F. (1995). A measured pace: Towards a philosophical understanding of the arts of the dance. Toronto: University of Toronto Press.

Stebbins, G. (1887). Delsarte system of expression. New York: Edgar S. Werner.

Straus, W. (1966). The upright posture. In M. Natson (Ed.), Essays in Phenomenology (pp. 169-192). Dordrecht: Springer, Science, Business Media, B.V.

Streader, T. \& Williams, A. (1985). Create your own stage lighting. London: Bell and Hyman Limited.

Toepfer, K. (1997). Empire of ecstasy: Nudity and movement in German body culture, 19101935. University of California Press.

Tözeren, A. (2000). Human body dynamics: Classical mechanics and human movement.New York: Springer-Verlag.

Vitrubius, M. (1826). The architecture of Marcus Vitrubius Pollio in ten books. Joseph Gwilt (Trad.). London: Priestly and Weale.

Weaver, J. (1721). Anatomical and mechanical: Lectures upon dancing. London: J. Brotherton and W. Meadows. 
Zollig, G. ( 2010). Searching for that "other land of dance": The phases in developing a choreography. In B. Bläsing, M. Puttke and T. Schack (Eds.), The neurocognition of dance: Mind, movement and motor skills (pp. 115-122). Hove: Psychology Press.

Data receção: 19/12/2019

Data aprovação: 25/05/2020 